\title{
Prospects of Future CMB Anisotropy Probes for Primordial Black Holes
}

\author{
Junsong Cang, ${ }^{a, b}$ Yu Gao, ${ }^{a}$ Yin-Zhe Ma ${ }^{c, d}$ \\ ${ }^{a}$ Key Laboratory of Particle Astrophysics, Institute of High Energy Physics, Chinese Academy \\ of Sciences, Beijing, 100049, China \\ ${ }^{b}$ School of Physical Sciences, University of Chinese Academy of Sciences, Beijing, 100049, \\ China \\ ${ }^{c}$ School of Chemistry and Physics, University of KwaZulu-Natal, Westville Campus, Private \\ Bag X54001, Durban, 4000, South Africa \\ ${ }^{d}$ NAOC-UKZN Computational Astrophysics Centre (NUCAC), University of KwaZulu-Natal, \\ Durban, 4000, South Africa \\ E-mail: cangjs@ihep.ac.cn, gaoyu@ihep.ac.cn, ma@ukzn.ac.za
}

\begin{abstract}
Cascade of particles injected as Hawking Radiation from Primordial Black Holes $(\mathrm{PBH})$ can potentially change the cosmic recombination history by ionizing and heating the intergalactic medium, which results in altering the anisotropy spectra of the Cosmic Microwave Background (CMB). In this paper, we study the expected sensitivity of several future CMB experiments in constraining the abundance of PBHs distributed in $10^{15} \sim 10^{17} \mathrm{~g}$ mass window according to four mass functions: the monochromatic, log-normal, power-law and critical collapse models. Our result shows that future experiments, such as CMB-S4 and PICO, can improve current Planck bounds by about two orders of magnitude. Most regions in PBH parameter space that are allowed by current CMB data, including monochromatically distributed PBHs with mass heavier than $3.8 \times 10^{16}$ grams, can be tested by upcoming missions with high significance.
\end{abstract}




\section{Contents}

1 Introduction 1

2 Hawking Radiation $\quad 2$

3 Energy injection and deposition $\quad 3$

4 PBH Mass Functions $\quad 4$

5 Impact on Recombination $\quad 6$

$\begin{array}{lll}6 & \text { Forecast Likelihood } & 7\end{array}$

$\begin{array}{lll}7 & \text { Results } & 7\end{array}$

8 Summary 12

$\begin{array}{lr}\text { A Experimental Specifications } & 13\end{array}$

\section{Introduction}

Conditions in the early universe may have led to the formation of Primordial Black Holes [1-5]. There are many mechanisms through which PBH can be created [1-12], including the primordial inhomogeneities [2] and collapse of domain wall bubbles [9], etc. Such black holes serve as an essential probe for the physics of the early universe and could manifest itself as cold dark matter (CDM) [13-15]. The allowed masses for PBH can span over a wide range from $10^{-38} \mathrm{M}_{\odot}$ to $10^{5} \mathrm{M}_{\odot}$ or higher [16], depending on the time of $\mathrm{PBH}$ formation after the Big Bang. PBHs in various mass ranges [15-21] can be probed by gravitational wave [22], lensing effect [23], emission from accretion of matter [18, 24, 25], CMB spectral distortions due to dissipation of small scale scalar perturbations [26-28], etc, see Ref.[29] for a recent review.

Hawking radiation is suitable to study the PBHs in the mass range of $\left[10^{15}, 10^{17}\right] \mathrm{g}[30-$ 33]. In this mass window the emissions are dominantly $e^{ \pm}, \gamma, \nu . e^{ \pm}$and $\gamma$ are electromagnetically interactive and deposit part of their kinetic energies into ionization of the intergalactic medium (IGM) after recombination, which increases the number density of free electrons and their scattering rates with CMB photons, leaving distinctive signatures on CMB anisotropy spectrum [34]. Fraction of the energy radiated from PBH can also be deposited to heat up IGM temperature or cause deviations in CMB energy spectra [35].

PBHs in this mass window can be further constrained by extragalactic photon background (EGB) [14, 16], diffuse supernova neutrino background [36, 37], galactic gamma-ray measurement [36, 38-42], etc. Currently the dominant constraint comes from the $21 \mathrm{~cm}$ measurement by EDGES $[43,44]$. However these constraints can suffer from huge uncertainties on background astrophysical models, most noticeably the galactic dark matter density profile and cosmic-ray propagation in our galaxy [38]. In comparison, bounds set by CMB anisotropy are more robust as most of the calculations involved are linear and based on well understood physics [34]. 
In this work we focus on the prospective PBH constraints from future CMB experiments. Over the past few years several experiments has been proposed to measure CMB anisotropy with higher precision than Planck, including satellite missions such as COrE [45, 46], LiteBIRD [47] and PICO [48-50], as well as ground-based experiments such as AdvACTPol [51], AliCPT [52, 53], Simons Array [54, 55], SPT-3G [56] and CMB-S4 [57, 58]. These experiments are expected to further strengthen current CMB bound on PBH set by Planck data, which has already been studied in a number of references $[17,32,33,59,60]$.

The structure of this paper is as follows: In Section 2 we review particle and energy emissions of Hawking radiation. Section 3 discusses the energy injection and deposition process for PBHs with monochromatic mass distribution, we then generalize our treatment to include PBHs with extended mass distributions in Section 4. Section 5 discusses the recombination history in the presence of $\mathrm{PBH}$ injection and the relevant impact on the CMB anisotropy spectrum. We outline our forecasting procedure in Section 6 and present our results in Section 7. Section 8 concludes this paper.

\section{Hawking Radiation}

The Hawking evaporation from a Schwarzschild black hole with mass $M$ is described by a thermal emission spectrum at a temperature [61]

$$
T_{\mathrm{PBH}}=\frac{1}{8 \pi G M}=1.06 \mathrm{TeV} \times\left(\frac{10^{10} \mathrm{~g}}{M}\right),
$$

where $G$ denotes the gravitational constant. The number of particles emitted per energy and time interval is given by $[61,62]$,

$$
\frac{\mathrm{d} N^{\alpha}}{\mathrm{d} \varepsilon \mathrm{d} t}=\frac{g^{\alpha}}{2 \pi} \frac{\Gamma_{\mathrm{s}}}{\mathrm{e}^{\varepsilon / T_{\mathrm{PBH}}}-(-1)^{2 s}},
$$

Here the superscript $\alpha$ labels the particle species, $g^{\alpha}$ is the degree of freedom for the particle, $s$ and $\varepsilon$ denote the spin and total energy of the emitted particle respectively, $\Gamma_{\mathrm{s}}$ is the absorption probability [16],

$$
\Gamma_{s}(M, \varepsilon)=\frac{\sigma_{s}(\varepsilon, M)}{\pi} \varepsilon^{2},
$$

where $\sigma_{\mathrm{s}}$ is the absorption cross section. At high energies where $\varepsilon \gg T_{\mathrm{PBH}}$ or equivalently $\varepsilon / T_{\mathrm{PBH}} \rightarrow \infty, \sigma_{\mathrm{s}}$ for all particle species approaches the geometric optics limit [16, 62],

$$
\sigma_{\mathrm{g}}=27 \pi G^{2} M^{2} .
$$

At lower energies $\sigma_{\mathrm{s}}$ is a function of $\varepsilon, M$ and $s$ and is solved numerically [62]. Here we adopt the $\sigma_{\mathrm{s}}$ given by Ref. [63] for $0 \leq \varepsilon / T_{\mathrm{PBH}} \leq 21$, and assume $\sigma_{\mathrm{s}}=\sigma_{\mathrm{g}}$ when $\varepsilon / T_{\mathrm{PBH}}>21$, which gives a reasonable approximation for the full $\sigma_{\mathrm{s}}$ function.

The mass loss rate caused by Hawking radiation of species $\alpha$ can be computed by,

$$
\dot{M}^{\alpha}=-\int \mathrm{d} \varepsilon \cdot \varepsilon \frac{\mathrm{d} N^{\alpha}}{\mathrm{d} \varepsilon \mathrm{d} t},
$$

which yields [64],

$$
\dot{M}^{\alpha}=-5.34 \times 10^{25} g^{\alpha} \omega^{\alpha}(M)\left(\frac{M}{\mathrm{~g}}\right)^{-2} \mathrm{~g} / \mathrm{s},
$$


In the relativistic limit [64], which corresponds to very light PBHs $\left(M \ll 10^{11} \mathrm{~g}\right)$,

$$
\begin{array}{ll}
\omega_{s=0}=0.267, \quad \omega_{s=1}=0.060, & \omega_{s=3 / 2}=0.022, \\
\omega_{s=2}=0.007, \quad \omega_{s=1 / 2}^{\nu}=0.147, & \omega_{s=1 / 2}^{e \pm}=0.142 .
\end{array}
$$

While thermal distribution has a high-energy tail, emissions of particles heavier than the $\mathrm{BH}$ temperature is exponentially suppressed. As a result, $\omega^{\alpha}$ for massive particle species decreases as black hole mass increases (lower $T_{\mathrm{PBH}}$ ). For electron and positron emissions, one can approximate $\omega^{\mathrm{e}^{ \pm}}$by $[33,59]$,

$$
\omega^{\mathrm{e}^{ \pm}}=0.142 \exp \left(-\frac{M}{9.4 \times 10^{16} \mathrm{~g}}\right) .
$$

In the PBH mass range we consider, the only particles that are emitted at appreciable amount are $\gamma, \nu, \mathrm{e}^{ \pm}$. As neutrinos are not electromagnetically interactive, hereafter we will restrict our discussions to the emission of $\gamma$ and $\mathrm{e}^{ \pm}$. It should be noted that although $\sum_{\alpha} \dot{M}^{\alpha}$ is nonzero, the resulting mass loss $\Delta M$ is negligible compared to $M$ in our mass range. Even for the most radiant PBH with $M=10^{15} \mathrm{~g}$, the fractional mass loss throughout the age of the universe is only at about $-\Delta M / M \sim 2 \%$. We will thus assume that PBH mass remains constant across the history of the universe.

\section{Energy injection and deposition}

In this section we will focus on PBHs with monochromatic $(\delta)$ mass distribution, for which the energy injection rate per unit volume by emission of particle of species $\alpha$ is given by,

$$
\left(\frac{\mathrm{d} E}{\mathrm{~d} V \mathrm{~d} t}\right)_{\mathrm{INJ}}^{\delta, \alpha}=-\dot{M}^{\alpha} n_{\mathrm{PBH}} .
$$

Here $\alpha=\left[e^{ \pm}, \gamma\right], n_{\mathrm{PBH}}$ is the number density of $\mathrm{PBH}$.

$$
n_{\mathrm{PBH}}=f_{\mathrm{PBH}} \frac{\Omega_{\mathrm{DM}} \rho_{\mathrm{cr}}(1+z)^{3}}{M}
$$

where $f_{\mathrm{PBH}} \equiv \Omega_{\mathrm{PBH}} / \Omega_{\mathrm{DM}}$ is the fraction of $\mathrm{DM}$ that consist of PBHs, $\rho_{\mathrm{cr}}$ is the critical density of the universe today.

Summing over contributions from all particle species gives the overall injection rate,

$$
\left(\frac{\mathrm{d} E}{\mathrm{~d} V \mathrm{~d} t}\right)_{\mathrm{INJ}}^{\delta}=\sum_{\alpha}\left(\frac{\mathrm{d} E}{\mathrm{~d} V \mathrm{~d} t}\right)_{\mathrm{INJ}}^{\delta, \alpha}=3.67 \times 10^{25} F_{\mathrm{PBH}} \Omega_{\mathrm{DM}} \rho_{\mathrm{cr}}(1+z)^{3} \mathrm{~s}^{-1},
$$

where,

$$
F_{\mathrm{PBH}} \equiv f_{\mathrm{PBH}} \frac{\sum_{\alpha}\left[g^{\alpha} \omega^{\alpha}\right]}{0.688}\left(\frac{M}{\mathrm{~g}}\right)^{-3}
$$

in which 0.688 is the relativistic value of $\sum_{\alpha}\left[g^{\alpha} \omega^{\alpha}\right]$.

After injection from $\mathrm{PBH}, e^{ \pm}$and $\gamma$ deposit their energies through a series of interactions with IGM and CMB photons. For distortions in CMB anisotropy, the most important deposition channels are: ionization (ion.) and excitation (exc.) of hydrogen and the heating 
of IGM (heat.). The rate of energy deposition into each channel (c) relates to the injection rate through a deposition efficiency $f_{\mathrm{c}}^{\alpha}$,

$$
\left(\frac{\mathrm{d} E}{\mathrm{~d} V \mathrm{~d} t}\right)_{\mathrm{DEP}, \mathrm{c}}^{\delta, \alpha}=f_{c}^{\alpha} \times\left(\frac{\mathrm{d} E}{\mathrm{~d} V \mathrm{~d} t}\right)_{\mathrm{INJ}}^{\delta, \alpha} .
$$

From the first principle, $f_{c}^{\alpha}$ can be constructed from the differential deposition efficiency given in Ref. [35]: $T_{\mathrm{c}, i j k}^{\alpha} \equiv T_{c}^{\alpha}\left(z_{i}, \varepsilon_{j}, z_{k}\right) \mathrm{d} \log (1+z)$. For a primary particle of species $\alpha$ injected at $z_{k}$ with energy $\varepsilon_{j}$, this quantity gives the fraction of $\varepsilon_{j}$ deposited into channel $\mathrm{c}$ at redshift $z_{i}$, during the time step corresponding to $\mathrm{d} \log (1+z)$. For a general energy injection spectrum, the relevant deposition efficiency is given by [35, 65],

$$
f_{c}^{\alpha}\left(z_{i}\right) \approx \frac{H\left(z_{i}\right)\left(1+z_{i}\right)^{3}}{\sum_{j} \varepsilon_{j} I^{\alpha}\left(z_{i}, \varepsilon_{j}\right) \mathrm{d} \varepsilon_{j}} \sum_{k} \frac{1}{\left(1+z_{k}\right)^{3} H\left(z_{k}\right)} \sum_{j} \varepsilon_{j} I^{\alpha}\left(z_{k}, \varepsilon_{j}\right) T_{\mathrm{c}, i j k}^{\alpha} \mathrm{d} \varepsilon_{j},
$$

where $I^{\alpha}(z, \varepsilon)$ describes the particle injection rate per unit $\varepsilon$ and unit volume,

$$
I^{\alpha}(z, \varepsilon) \equiv \frac{\mathrm{d} N^{\alpha}}{\mathrm{d} \varepsilon \mathrm{d} t \mathrm{~d} V}(z, \varepsilon)=\frac{\mathrm{d} N^{\alpha}}{\mathrm{d} \varepsilon \mathrm{d} t} \times n_{\mathrm{PBH}} .
$$

The overall PBH deposition rate is given by summing over all particle species,

$$
\left(\frac{\mathrm{d} E}{\mathrm{~d} V \mathrm{~d} t}\right)_{\mathrm{DEP}, \mathrm{c}}^{\delta}=\sum_{\alpha}\left(\frac{\mathrm{d} E}{\mathrm{~d} V \mathrm{~d} t}\right)_{\mathrm{DEP}, \mathrm{c}}^{\delta, \alpha}=f_{\mathrm{c}} \times\left(\frac{\mathrm{d} E}{\mathrm{~d} V \mathrm{~d} t}\right)_{\mathrm{INJ}}^{\delta}
$$

where $f_{\mathrm{c}}$ is an overall deposition efficiency,

$$
f_{\mathrm{c}} \equiv \frac{\sum_{\alpha} g^{\alpha} \omega^{\alpha} f_{\mathrm{c}}^{\alpha}}{\sum_{\alpha} g^{\alpha} \omega^{\alpha}}=\frac{4 \omega^{\mathrm{e}^{ \pm}} f_{\mathrm{c}}^{\mathrm{e}^{ \pm}}+2 \omega^{\gamma} f_{\mathrm{c}}^{\gamma}}{4 \omega^{\mathrm{e}^{ \pm}}+2 \omega^{\gamma}} .
$$

\section{PBH Mass Functions}

Many of the existing PBH constraints in the literatures [32, 38, 40, 60] normally include monochromatic mass distribution. Although such distribution model is theoretically viable [66], many other $\mathrm{PBH}$ formation scenarios tend to predict extended mass distributions [67-75], which can generally be described by a PBH mass function,

$$
\Psi(M) \equiv \frac{1}{f_{\mathrm{PBH}} \rho_{\mathrm{DM}}} \frac{\mathrm{d} \rho_{\mathrm{PBH}}(M)}{\mathrm{d} M} .
$$

A monochromatic mass spectrum is simply,

$$
\Psi\left(M^{\prime}\right)=\delta_{\mathrm{D}}\left(M-M^{\prime}\right) .
$$

We will also consider three types of extended PBH mass functions:

- Log-normal model $[67,73,74]$

$$
\Psi(M)=\frac{1}{\sqrt{2 \pi} \sigma M} \exp \left(-\frac{\left(\log \left[M / M_{c}\right]\right)^{2}}{2 \sigma^{2}}\right) .
$$




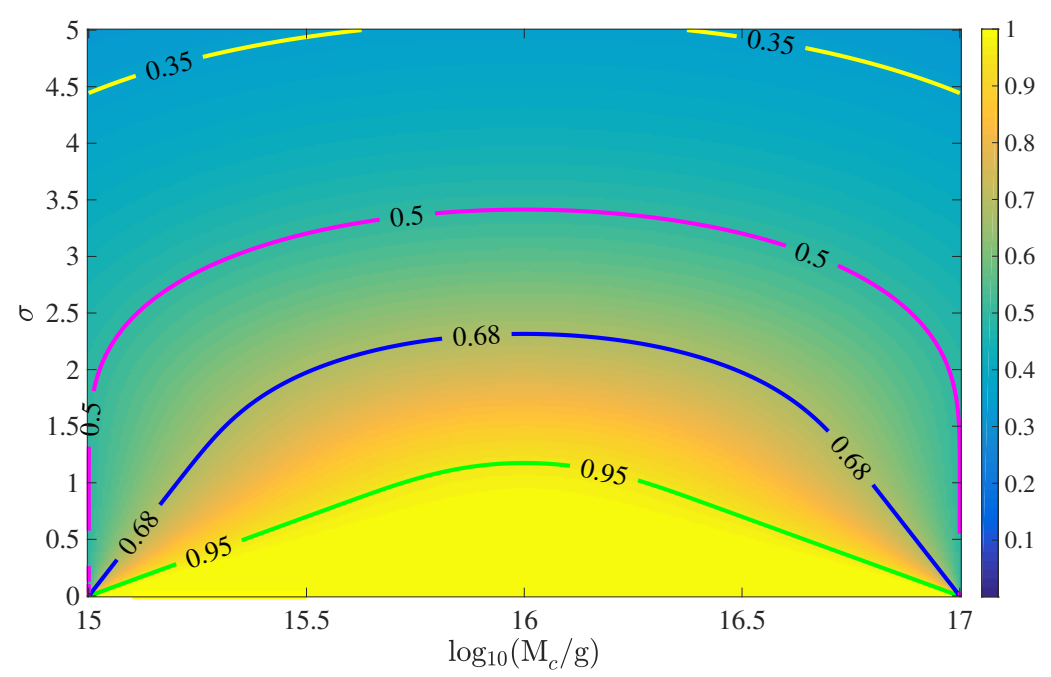

Figure 1. Fraction of PBHs density contained in $10^{15} \mathrm{~g} \sim 10^{17} \mathrm{~g}$ mass range for log-normal model, given by $\int_{10^{15} \mathrm{~g}}^{10^{17} \mathrm{~g}} \mathrm{~d} M \Psi$.

- Critical Collapse model [71-73]

$$
\Psi(M) \propto M^{2.85} \exp \left(-\left(M / M_{c}\right)^{2.85}\right) .
$$

- Power-law model [73]

$$
\Psi(M) \propto M^{\gamma-1}\left(M_{\min }<M<M_{\max }\right) .
$$

In general, the log-normal model can provide a good approximation for many other distributions and is theoretically motivated in multiple $\mathrm{PBH}$ formation scenarios [67, 70, 73-75]. The Critical Collapse model is possible if the density fluctuation leading to PBHs formation has a $\delta$ function power spectrum, whereas PBHs produced from scale-invariant power spectrum can have a power-law distribution [73]. Note that the power law index $\gamma$ in $\mathrm{Eq}(4.5)$ is given by $\gamma=-2 \omega /(1+\omega)$, where $\omega$ is the equation of state parameter at the epoch during which PBHs formed. Here we only consider post-inflation PBH formation in which $\gamma$ ranges in $[-1,1]$. In this context $(\gamma<1)$, the power-law $\Psi(M)$ peaks at $M_{\min }$ and becomes more concentrated as $\gamma$ decreases, so $\gamma$ serves as a good indicator of the distribution width of $\Psi(M)$. Special treatment is required for $\gamma=0$, which corresponds to PBHs formation during matter-dominated phase of the universe. We will ignore this scenario and refer interested readers to Ref.[76] for detailed analysis.

With these generalizations, the total deposition rate now takes the form,

$$
\left(\frac{\mathrm{d} E}{\mathrm{~d} V \mathrm{~d} t}\right)_{\mathrm{DEP}, \mathrm{c}}=\int_{10^{15} \mathrm{~g}}^{10^{17} \mathrm{~g}} \mathrm{~d} M \cdot \Psi(M)\left(\frac{\mathrm{d} E}{\mathrm{~d} V \mathrm{~d} t}(M)\right)_{\mathrm{DEP}, \mathrm{c}}^{\delta} .
$$

Note that our mass functions are normalized for $M \in[0, \infty]$, whereas $\operatorname{Eq}(4.6)$ only account for holes with $M \in\left[10^{15} \mathrm{~g}, 10^{17} \mathrm{~g}\right]$, this could overestimate PBH abundance if a significant amount 
of PBH density were distributed outside this mass window. The critical collapse mass function is very sharp and can be well approximated by a semi-monochromatic log-normal distribution with $\sigma=0.26$ [73], so it is safe from this problem as long as $M_{c}$ is not very close to our cut-off masses. This issue can also be avoided in power-law model by enforcing $M_{\min } \geq 10^{15} \mathrm{~g}$ and $M_{\max } \leq 10^{17} \mathrm{~g}$. However as shown in Fig.1, the log-normal mass function is susceptible to this problem, especially for very large $\sigma$ values. Therefore for log-normal distributions with

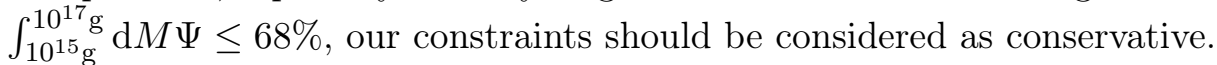

\section{Impact on Recombination}

In presence of $\mathrm{PBH}$ injection, the recombination equations for ionization fraction $x_{\mathrm{e}}$ and matter temperature $T_{\text {IGM }}$ becomes $[77,78]$

$$
\begin{gathered}
\frac{\mathrm{d} x_{\mathrm{e}}}{\mathrm{d} t}=\left(\frac{\mathrm{d} x_{\mathrm{e}}}{\mathrm{d} t}\right)_{0}+I_{\text {ion }}+I_{\mathrm{exc}}, \\
\frac{\mathrm{d} T_{\mathrm{IGM}}}{\mathrm{d} t}=\left(\frac{\mathrm{d} T_{\mathrm{IGM}}}{\mathrm{d} t}\right)_{0}+\frac{2 Q_{\text {heat }}}{3\left(1+f_{\mathrm{He}}+x_{\mathrm{e}}\right)} .
\end{gathered}
$$

Here the standard evolution equations without additional energy injection are labeled with the subscript $0[78,79], f_{\mathrm{He}}$ is the helium fraction by number of nuclei. $I_{\mathrm{ion}}$ and $I_{\mathrm{exc}}$ describe the hydrogen ionization from ground state and excited $n=2$ state respectively,

$$
\begin{aligned}
I_{\text {ion }}(z) & =\frac{1}{n_{\mathrm{H}}(z) E_{i}}\left(\frac{\mathrm{d} E}{\mathrm{~d} V \mathrm{~d} t}\right)_{\mathrm{DEP}, \text { ion }} \\
I_{\mathrm{exc}}(z) & =\frac{1-C}{n_{\mathrm{H}}(z) E_{\alpha}}\left(\frac{\mathrm{d} E}{\mathrm{~d} V \mathrm{~d} t}\right)_{\mathrm{DEP}, \mathrm{exc}},
\end{aligned}
$$

where $E_{i}=13.6 \mathrm{eV}, E_{\alpha}=10.2 \mathrm{eV}$. $n_{\mathrm{H}}$ is the number density of hydrogen nuclei, $C$ is the probability for a $n=2$ hydrogen atom to transit back to $n=1$ ground state before getting ionized [78]. $Q_{\text {heat }}$ is the IGM heating rate induced by $\mathrm{PBH}$,

$$
Q_{\text {heat }}(z)=\frac{1}{n_{\mathrm{H}}(z)}\left(\frac{\mathrm{d} E}{\mathrm{~d} V \mathrm{~d} t}\right)_{\text {DEP,heat }} .
$$

It should be noted that, after the heating by PBHs' radiation, the raised IGM temperature means that collisional ionization might become more manifest at certain redshifts [80-82]. This issue is beyond the scope of this paper and is not included in our numerical process.

$\mathrm{CMB}$ can provide a powerful probe for the ionizing effect of $\mathrm{PBH}$, which increases the ionization fraction and thereby enhancing scattering rate between CMB photons and free electrons. Such effect can leave fingerprints on CMB anisotropy by damping small scale temperature and polarization correlations while shifting peak locations for polarization anisotropy spectra [34, 83]. The PBH heating term can increase gas temperature and be constrained through experiments that measure the $21 \mathrm{~cm}$ emission line from neutral hydrogen $[30,43,84-$ 86]. 


\section{Forecast Likelihood}

At this forecast we use temperature and E-mode polarization correlations. Foreground contamination is assumed to have been fully removed and we only use frequency channels between 89 and $160 \mathrm{GHz}$, which roughly corresponds to the frequency range where CMB signal is expected to have the highest intensity compared to foreground components such as synchrotron and dust [87]. Our mock data $\hat{C}_{\ell}$ can be expressed as a combination of fiducial CMB signal $\bar{C}_{\ell}$ and experimental noise $N_{\ell}$,

$$
\begin{gathered}
\hat{C}_{\ell}=\bar{C}_{\ell}+N_{\ell} \\
\bar{C}_{\ell} \equiv\left[\begin{array}{cc}
\bar{C}_{\ell}^{T T} & \bar{C}_{\ell}^{T E} \\
\bar{C}_{\ell}^{T E} & \bar{C}_{\ell}^{E E}
\end{array}\right], N_{\ell} \equiv\left[\begin{array}{cc}
N_{\ell}^{T T} & 0 \\
0 & N_{\ell}^{E E}
\end{array}\right],
\end{gathered}
$$

here $\bar{C}_{\ell} s$ are generated by CAMB [88] codes for a flat $\Lambda$ CDM cosmology set by Planck 2018 data [89]: $\Omega_{\mathrm{b}} h^{2}=0.02242, \Omega_{\mathrm{c}} h^{2}=0.11933,100 \theta_{\mathrm{MC}}=1.04101, \tau=0.0561, \log \left(10^{10} A_{\mathrm{s}}\right)=$ $3.047, n_{\mathrm{s}}=0.9665$. $N_{\ell}$ is given by [90],

$$
\begin{aligned}
& N_{\ell}^{\mathrm{EE}}=\left[\sum_{\nu} \frac{1}{N_{\ell, \nu}^{\mathrm{EE}}}\right]^{-1}, N_{\ell}^{\mathrm{TT}}=N_{\ell}^{\mathrm{EE}} / 2, \\
& N_{\ell, \nu}^{\mathrm{EE}}=\delta P_{\nu}^{2} \exp \left[\ell(\ell+1) \frac{\theta_{\mathrm{FWHM}, \nu}^{2}}{8 \ln 2}\right],
\end{aligned}
$$

where $\delta P_{\nu}$ denotes the instrumental white noise level for frequency $\nu$, measured in $\mu \mathrm{k} \cdot \mathrm{rad}$. $\theta_{\mathrm{FWHM}, \nu}$ is the Full-Width at Half-Minimum beam size in radians. For an experiment with a sky coverage of $f_{\text {sky }}$, the effective $\chi^{2}$ for the likelihood used in our analysis can be written as [91],

$$
\chi_{\text {eff }}^{2} \equiv-2 \ln \mathcal{L}=f_{\text {sky }} \sum_{\ell}(2 \ell+1)\left[\operatorname{Tr}\left(\hat{C}_{\ell} C_{\ell}^{-1}\right)-\log \left|\hat{C}_{\ell} C_{\ell}^{-1}\right|-2\right]
$$

where $C_{\ell}$, given by

$$
C_{\ell} \equiv\left[\begin{array}{ll}
C_{\ell}^{T T} & C_{\ell}^{T E} \\
C_{\ell}^{T E} & C_{\ell}^{E E}
\end{array}\right],
$$

is calculated by CAMB for each sampled point in the parameter space for a " $\Lambda \mathrm{CDM}+\mathrm{PBH}$ " cosmology. Specifications for experiments considered in our forecast can be found in Table 2.

\section{$7 \quad$ Results}

We use modified HyRec [79] and CAMB [88] codes to solve for recombination history and resulting CMB anisotropy spectrum. In addition to forecasted results for future experiments, we also show constraints set by Planck 2018 data [92]: (i) the plik-lite TTTEEE likelihood for $\ell>29$, (ii) the low- $\ell$ TT and EE likelihoods for $\ell<29$, (iii) lensing likelihood. All monochromatic constraints, shown in Fig.2 and the top left panel in Fig.3, are obtained by sampling our likelihoods using CosmoMC [93, 94]. The six $\Lambda$ CDM parameters are varied in MCMC analysis and marginalized in our results. 


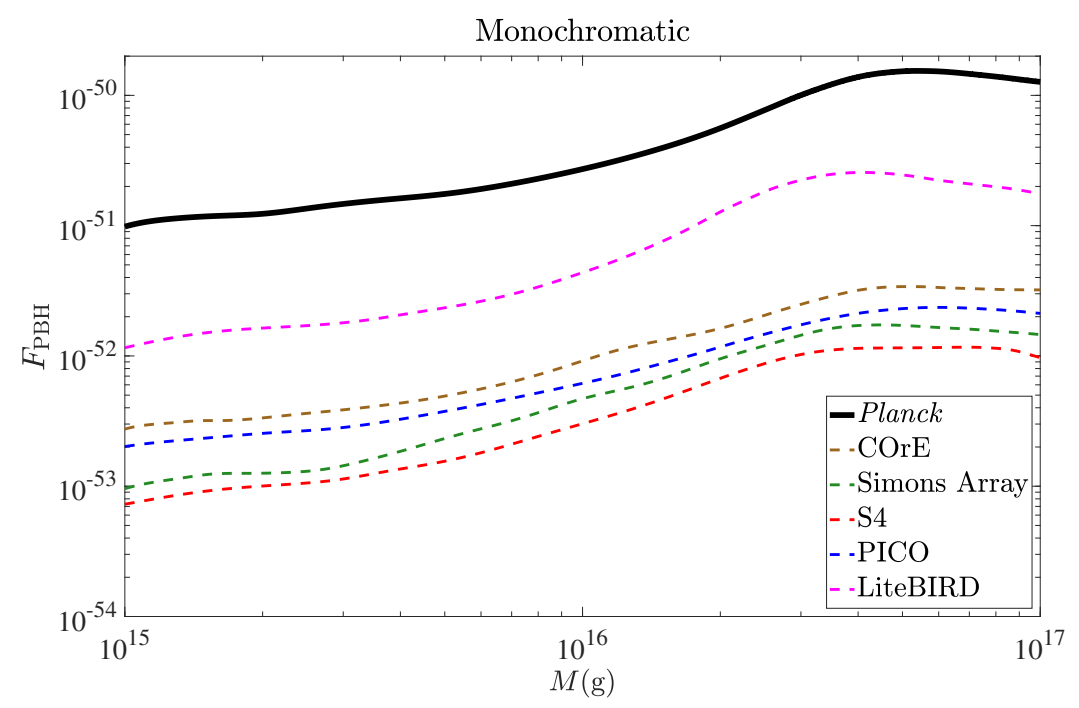

Figure 2. Prospective $95 \%$ C.L upper bounds on $F_{\mathrm{PBH}}$ defined in Eq.(3.4).

For computational convenience, here we calculate $f_{\mathrm{PBH}}$ upper bounds for extended distributions using [73, 95]

$$
f_{\mathrm{PBH}} \leq 1 / \int_{10^{15} \mathrm{~g}}^{10^{17} \mathrm{~g}} \mathrm{~d} M \frac{\Psi(M)}{f_{\max }(M)},
$$

where $f_{\max }(M)$ is our MCMC bounds on $f_{\mathrm{PBH}}$ for the monochromatic PBHs with mass $M$. This method was proven exact provided that $f_{\max }(M)$ were derived from one single observable[73]. For all our crosschecks with MCMC results for Planck, which has also been included in Fig.3 in black solid lines, we found that constraints derived using this equation agrees very well with that given by full MCMC simulation (at $10 \%$ level).

As shown in Figs.3 and 4, for all PBH distributions we considered, significantly improved $f_{\mathrm{PBH}}$ constraints are expected from future CMB observations. For monochromatic and extended mass functions, there are still regions in parameter space allowed by current data where $\mathrm{PBH}$ can serve as the dominant $\left(f_{\mathrm{PBH}} \sim 1\right)$ DM component. For example, monochromatic PBHs heavier than $3.8 \times 10^{16} \mathrm{~g}$ can still account for all DM in the universe according to Planck. Future missions are capable of testing all these possibilities. The Simons Array $[54,55]$, which has already started taking data, along with proposed missions such as PICO [48, 50] and CMB-S4 [57, 58], can constrain monochromatic PBH abundance down to $F_{\mathrm{PBH}} \sim 10^{-53}$, improved by about two orders of magnitudes compared with Planck, and about one order of magnitude more stringent than the EBG bounds [14, 16]. We also find that our monochromatic Planck bound is in good agreement with that in [59].

In general, as lighter PBHs are more radiant, $f_{\mathrm{PBH}}$ constraints weakens as $M$ increases. By choosing $F_{\mathrm{PBH}}$ to parameterize monochromatic $\mathrm{PBH}$ injection instead of $f_{\mathrm{PBH}}$, we factored out the dependence on the $M^{3}$ term and massive particle emission fraction $\left(\omega^{\mathrm{e}^{ \pm}}(M)\right)$ in Eq.(3.3). As a result, the dependence of $F_{\mathrm{PBH}}$ constraints on PBH mass in Fig.2 is determined mostly by the way in which the deposition efficiency relates to $M$. A visible peak on $F_{\mathrm{PBH}}$ can be seen at around $4 \times 10^{16} \mathrm{~g}$, which roughly corresponds to regions where the deposition efficiency is at the lowest. 

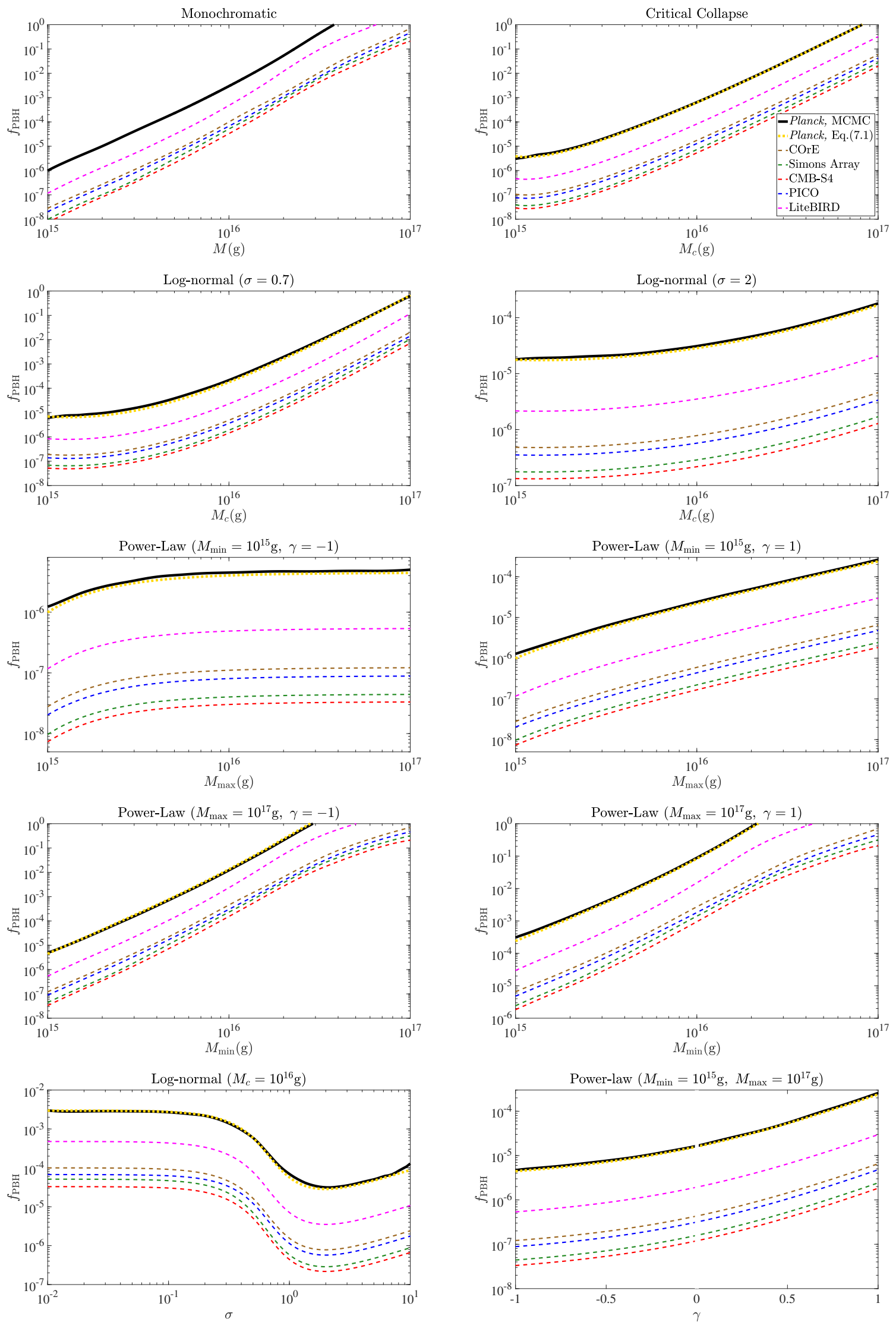

Figure 3. Prospective $95 \%$ C.L upper bounds on $f_{\mathrm{PBH}}$, legend applies to all panels. For extended distributions, Planck constraints derived from MCMC analysis and Eq. (7.1) are shown in black solid and yellow dotted lines respectively. 

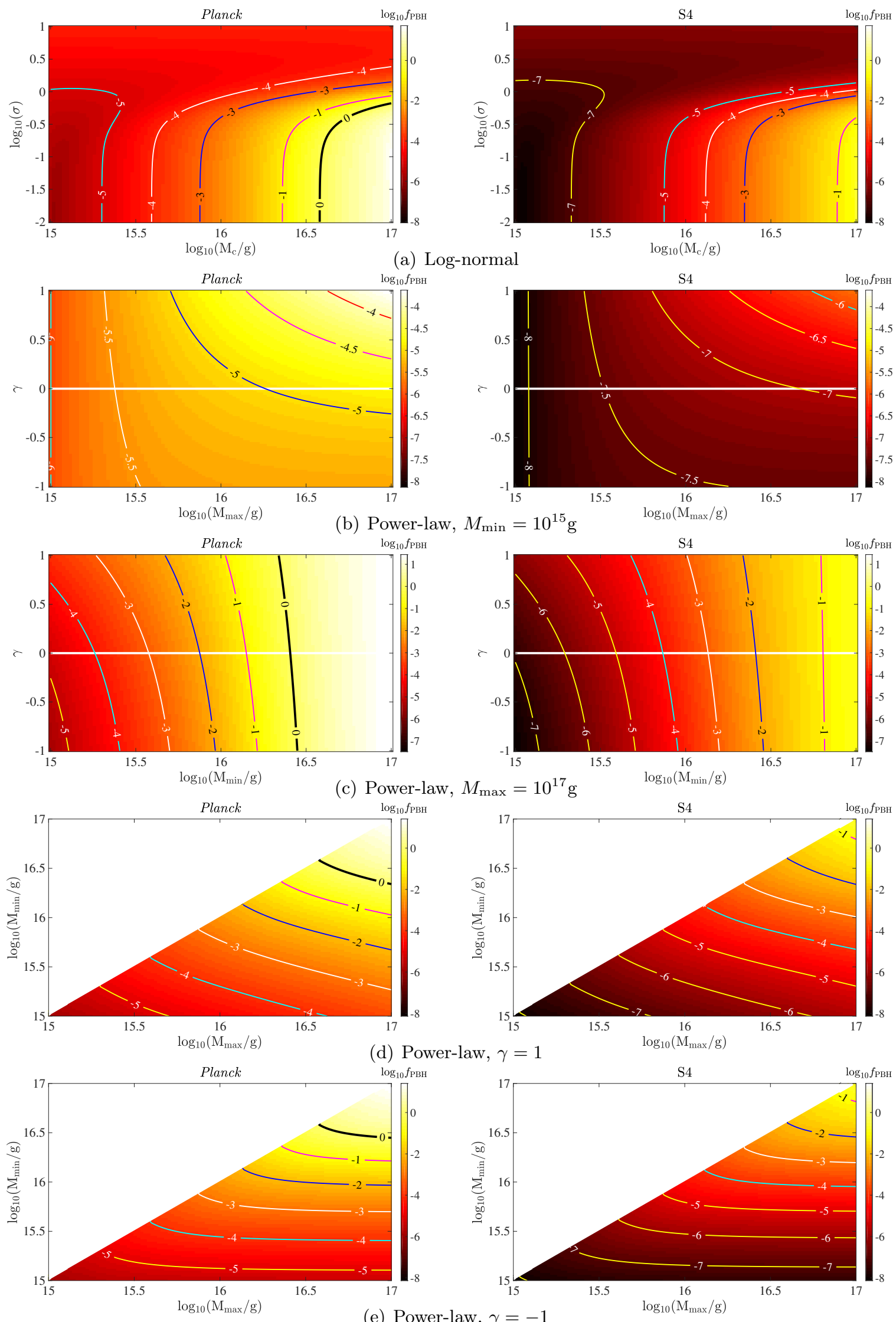

(e) Power-law, $\gamma=-1$

Figure 4. Planck (left) and projected CMB-S4 (right) 95\% C.L upper bounds on $\log _{10} f_{\mathrm{PBH}}$. Black contours indicates regions where PBHs can account for all of DM. The white horizontal lines in panels $\mathrm{b}$ and $\mathrm{c}$ indicate $\gamma=0$, which is not considered in this work. 
For monochromatic PBHs, the EGB upper bound on $f_{\mathrm{PBH}}$ can be parameterized by[14],

$$
f^{\mathrm{EGB}}=2 \times 10^{-8}\left(\frac{M}{M_{\star}}\right)^{3+\epsilon}\left(M_{\star}<M<10^{18} \mathrm{~g}\right),
$$

where $M_{\star}=5 \times 10^{14} \mathrm{~g}$ is the mass of a PBH whose lifetime equals the age of the Universe. $\epsilon$ parameterizes the spectral index of extragalactic intensity and has values between 0.1 and 0.4. Similarly we found that our CMB bound can be well approximated by

$$
f^{\mathrm{CMB}}=\frac{4.32}{K} \times 10^{-8}\left(\frac{M}{M_{\star}}\right)^{3+\epsilon^{\prime}}\left(10^{15} \mathrm{~g}<M<10^{17} \mathrm{~g}\right),
$$

where $\epsilon^{\prime}=0.84, K$ is an experiment specific scaling factor that describes how stringent each mission constrains $f_{\mathrm{PBH}} . \quad K$ is normalized to unity for Planck and is generally larger for forecasted missions. The prospective value of this scaling factor for future experiments are listed in Table.1. The experiments' specifications are listed in Appendix A.

\begin{tabular}{c|c}
\hline Experiment & Scaling Factor \\
\hline Planck & 1 \\
COrE & 37 \\
CMB-S4 & 113 \\
PICO & 53 \\
LiteBIRD & 7 \\
Simons Array & 80 \\
\hline
\end{tabular}

Table 1. The best-fit scaling factor $K$ defined in Eq. (7.3), for experimental specifics and $\ell$ ranges listed in Tab 2.

As expected, we recover the monochromatic constraints when $\sigma \rightarrow 0$ in log-normal model or $M_{\min } \rightarrow M_{\max }$ in power-law model. For the log-normal distribution, $\sigma=0.2$ can already give a good approximation for monochromatic $f_{\mathrm{PBH}}$ bound, with an average deviation of about $27 \%$. We find that so long as the majority $(\geq 68 \%)$ of PBHs density are covered in our targeted $\left[10^{15}, 10^{17}\right] \mathrm{g}$ mass range, increasing the distribution width $\sigma$ in log-normal model tightens $f_{\mathrm{PBH}}$ constraints, which is in agreement with the claims in Refs.[73]. PBHs with $\sigma$ larger than 0.66 are completely ruled out as the dominant DM component by Planck data. However for regions where an appreciable amount of PBH density are distributed outside the $\left[10^{15}, 10^{17}\right] \mathrm{g}$ window, $f_{\mathrm{PBH}}$ bound relaxes as we increase $\sigma$. For power-law distribution, we find that higher $\gamma$, which corresponds to wider distributions, allows for higher $f_{\mathrm{PBH}}$. For $\gamma=1$, the power-law mass function automatically reduces to an uniform distribution in $\left[M_{\min }, M_{\max }\right]$ with $\Psi=$ Const, results for which are also presented in Figs. 3 and 4.

Note that in case of null-signal, whether extending the PBH distribution tighten the $f_{\mathrm{PBH}}$ upper bounds depends on whether the extension leads to more $\mathrm{PBH}$ radiation. For the log-normal model, if the parameters in the mass function still ensure that a significant fraction of $\mathrm{PBH}$ density is contained in the mass range that can be probed through Hawking radiation, then increasing the distribution width will continuously include smaller, more radiant PBHs, which in turn tightens $f_{\mathrm{PBH}}$ bounds. Otherwise if the majority of PBHs were distributed outside the probed mass window, then the extension will include less $\mathrm{PBH}$ radiation and the constraints on $f_{\mathrm{PBH}}$ will be relaxed, making it possible to evade monochromatic bounds by merely extending the distribution. 


\section{Summary}

Hawking radiation from primordial black holes during the cosmic dark ages can ionize and heat the intergalactic medium. This effect increases the scattering between CMB photons and free electrons, thereby changing the CMB anisotropy spectrum. In this paper we forecast the sensitivity of several future CMB experiments in constraining PBHs in $10^{15} \sim 10^{17}$ g mass range. We find that future experiments can significantly improve current CMB bounds and rule out PBHs as the dominant DM component for all mass functions considered. For the conventional monochromatic distribution, LiteBIRD constrains $f_{\mathrm{PBH}} / M^{3}$ to orders of $10^{-52} \mathrm{~g}^{-3}$, bounds from COrE, PICO, Simons Array and CMB-S4 can extend down to $f_{\mathrm{PBH}} / M^{3} \sim 10^{-53} \mathrm{~g}^{-3}$, improved by about two orders of magnitudes compared to Planck. We also considered three extended distribution models: log-normal, power-law and critical collapse. Provided that at least $68 \%$ of PBH density are still contained in $\left[10^{15}, 10^{17}\right] \mathrm{g}$, for log-normal models with large distribution width $\sigma$ and the critical collapse distribution, $f_{\mathrm{PBH}}$ bound tightens relative to the monochromatic results. Most of our extended constraints are obtained by re-interpreting the monochromatic bounds, and we have cross-checked that these results are consistent with that derived from full MCMC analysis.

Acknowledgements J.C. is supported by the Sino US Cooperation Project of Ministry of Science and Technology (No. 2016YFE0104700). Y.G. is partially supported by the Institute of High Energy Physics, Chinese Academy of Sciences, under the grant no. Y7515560U1. YZM acknowledges National Research Foundation of South Africa with grant no. NRF-120385, NRF-120378, NRF-109577, and National Science Foundation of China with grant no. NSFC-11828301. 


\section{A Experimental Specifications}

\begin{tabular}{|c|c|c|c|c|c|c|}
\hline Experiment & $f_{\text {sky }}$ & $\ell_{\min }$ & $\ell_{\max }$ & $\begin{array}{c}\nu \\
(\mathrm{GHz})\end{array}$ & $\begin{array}{c}\delta P \\
(\mu \mathrm{K}-\operatorname{arcmin})\end{array}$ & $\begin{array}{c}\theta_{\text {FWHM }} \\
\text { (arcmin) }\end{array}$ \\
\hline \multirow{6}{*}{ COrE $[45,46]$} & \multirow{6}{*}{0.7} & \multirow{6}{*}{2} & \multirow{6}{*}{3000} & 90 & 7.3 & 12.1 \\
\hline & & & & 100 & 7.1 & 10.9 \\
\hline & & & & 115 & 7.0 & 9.6 \\
\hline & & & & 130 & 5.5 & 8.5 \\
\hline & & & & 145 & 5.1 & 7.7 \\
\hline & & & & 160 & 5.2 & 7.0 \\
\hline \multirow{2}{*}{ CMB-S4 $[57,58]$} & \multirow{2}{*}{0.62} & \multirow{2}{*}{30} & \multirow{2}{*}{3000} & 95 & 2.9 & 2.2 \\
\hline & & & & 145 & 2.8 & 1.4 \\
\hline \multirow{4}{*}{ PICO [48-50] } & \multirow{4}{*}{0.7} & \multirow{4}{*}{2} & \multirow{4}{*}{4000} & 90 & 2.1 & 9.5 \\
\hline & & & & 108 & 1.7 & 7.9 \\
\hline & & & & 129 & 1.5 & 7.4 \\
\hline & & & & 155 & 1.3 & 6.2 \\
\hline \multirow{4}{*}{ LiteBIRD [47] } & \multirow{4}{*}{0.7} & \multirow{4}{*}{2} & \multirow{4}{*}{200} & 89 & 11.7 & 35 \\
\hline & & & & 100 & 9.2 & 29 \\
\hline & & & & 119 & 7.6 & 25 \\
\hline & & & & 140 & 5.9 & 23 \\
\hline \multirow{2}{*}{ Simons Array $[54,55]$} & \multirow{2}{*}{0.65} & \multirow{2}{*}{30} & \multirow{2}{*}{3000} & 95 & 13.9 & 5.2 \\
\hline & & & & 150 & 11.4 & 3.5 \\
\hline
\end{tabular}

Table 2. Design specifications for experiments considered in our forecast. We only list frequency channels $(89 \mathrm{GHz} \sim 160 \mathrm{GHz})$ used in our simulation. $f_{\text {sky }}$ and $\ell_{\min }$ for space-borne missions are set to 0.7 and 2 respectively. Note that the constraints may improve if the $\ell$ ranges are further optimized with each experiment's realistic noise model. For example, PICO constraint improves by $53 \%$ when using $\ell$ range of $[30,3000]$ with our noise estimate in Eq. 6.4.

\section{References}

[1] I.D. Novikov, The hypothesis of cores retarded during expansion and the hot cosmological model, Astronomicheskii Zhurnal 10 (1967) 602.

[2] S. Hawking, Gravitationally Collapsed Objects of Very Low Mass, Monthly Notices of the Royal Astronomical Society 152 (1971) 75.

[3] B.J. Carr, The primordial black hole mass spectrum, Astrophysical Journal 201 (1975) 1.

[4] V. Canuto, On the origin of hawking mini black-holes and the cold early universe, Mon. Not. Roy. Astron. Soc. 184 (1978) 721.

[5] I.D. Novikov, A.G. Polnarev, A.A. Starobinskii and I.B. Zeldovich, Primordial black holes, Astron. Astroph 80 (1979) 104.

[6] S. Hawking and I. Moss, Supercooled phase transitions in the very early universe, Physics Letters B 110 (1982) 35 .

[7] S. Hawking, Gravitational radiation from collapsing cosmic string loops, Physics Letters B $\mathbf{2 4 6}$ (1990) 36 . 
[8] E. Bugaev and P. Klimai, Large curvature perturbations near horizon crossing in single-field inflation models, Phys. Rev. D 78 (2008) 063515.

[9] J. Liu, Z.-K. Guo and R.-G. Cai, Primordial Black Holes from Cosmic Domain Walls, Phys. Rev. D 101 (2020) 023513 [1908.02662].

[10] T.-J. Gao and Z.-K. Guo, Primordial Black Hole Production in Inflationary Models of Supergravity with a Single Chiral Superfield, Phys. Rev. D 98 (2018) 063526 [1806.09320].

[11] M.Y. Khlopov, Primordial Black Holes, Res. Astron. Astrophys. 10 (2010) 495 [0801.0116].

[12] K.M. Belotsky, V.I. Dokuchaev, Y.N. Eroshenko, E.A. Esipova, M.Y. Khlopov, L.A. Khromykh et al., Clusters of primordial black holes, Eur. Phys. J. C 79 (2019) 246 [1807.06590].

[13] G. Chapline, Cosmological effects of primordial black holes, Nature 253 (1975) 251.

[14] B. Carr, F. Kuhnel and M. Sandstad, Primordial Black Holes as Dark Matter, Phys. Rev. D 94 (2016) 083504 [1607.06077].

[15] A. Kashlinsky et al., Electromagnetic probes of primordial black holes as dark matter, 1903.04424.

[16] B. Carr, K. Kohri, Y. Sendouda and J. Yokoyama, New cosmological constraints on primordial black holes, Phys. Rev. D 81 (2010) 104019 [0912.5297].

[17] V. Poulin, J. Lesgourgues and P.D. Serpico, Cosmological constraints on exotic injection of electromagnetic energy, JCAP 03 (2017) 043 [1610.10051].

[18] Y. Ali-Haïmoud and M. Kamionkowski, Cosmic microwave background limits on accreting primordial black holes, Phys. Rev. D 95 (2017) 043534 [1612.05644].

[19] P. Montero-Camacho, X. Fang, G. Vasquez, M. Silva and C.M. Hirata, Revisiting constraints on asteroid-mass primordial black holes as dark matter candidates, JCAP 08 (2019) 031 [1906. 05950].

[20] A. Arbey, J. Auffinger and J. Silk, Constraining primordial black hole masses with the isotropic gamma ray background, Phys. Rev. D 101 (2020) 023010 [1906.04750].

[21] T.N. Ukwatta, D. Stump, J. Linnemann, S.S. Marinelli, T. Yapici, K. Tollefson et al., Observational Characteristics of the Final Stages of Evaporating Primordial Black Holes, PoS ICRC2015 (2016) 793 [1507.01648].

[22] Z.-C. Chen, C. Yuan and Q.-G. Huang, Pulsar Timing Array Constraints on Primordial Black Holes with NANOGrav 11-Year Dataset, Phys. Rev. Lett. 124 (2020) 251101 [1910.12239].

[23] K. Griest, A.M. Cieplak and M.J. Lehner, New limits on primordial black hole dark matter from an analysis of kepler source microlensing data, Phys. Rev. Lett. 111 (2013) 181302.

[24] M. Ricotti, J.P. Ostriker and K.J. Mack, Effect of primordial black holes on the cosmic microwave background and cosmological parameter estimates, The Astrophysical Journal $\mathbf{6 8 0}$ (2008) 829.

[25] H. Tashiro and N. Sugiyama, The effect of primordial black holes on $21 \mathrm{~cm}$ fluctuations, Mon. Not. Roy. Astron. Soc. 435 (2013) 3001 [1207.6405].

[26] K. Kohri, T. Nakama and T. Suyama, Testing scenarios of primordial black holes being the seeds of supermassive black holes by ultracompact minihalos and CMB $\mu$-distortions, Phys. Rev. D 90 (2014) 083514 [1405.5999].

[27] T. Nakama, B. Carr and J. Silk, Limits on primordial black holes from $\mu$ distortions in cosmic microwave background, Phys. Rev. D 97 (2018) 043525 [1710.06945].

[28] J. Chluba, A.L. Erickcek and I. Ben-Dayan, Probing the Inflaton: Small-scale Power Spectrum Constraints from Measurements of the Cosmic Microwave Background Energy Spectrum, ApJ 758 (2012) 76 [1203.2681]. 
[29] B. Carr and F. Kuhnel, Primordial Black Holes as Dark Matter: Recent Developments, 2006.02838.

[30] K.J. Mack and D.H. Wesley, Primordial black holes in the Dark Ages: Observational prospects for future 21cm surveys, 0805.1531.

[31] H. Tashiro and N. Sugiyama, Constraints on primordial black holes by distortions of the cosmic microwave background, Phys. Rev. D 78 (2008) 023004.

[32] S. Clark, B. Dutta, Y. Gao, L.E. Strigari and S. Watson, Planck Constraint on Relic Primordial Black Holes, Phys. Rev. D 95 (2017) 083006 [1612.07738].

[33] H. Poulter, Y. Ali-Haïmoud, J. Hamann, M. White and A.G. Williams, CMB constraints on ultra-light primordial black holes with extended mass distributions, 1907.06485.

[34] N. Padmanabhan and D.P. Finkbeiner, Detecting dark matter annihilation with CMB polarization: Signatures and experimental prospects, Phys. Rev. D 72 (2005) 023508 [astro-ph/0503486].

[35] T.R. Slatyer, Indirect Dark Matter Signatures in the Cosmic Dark Ages II. Ionization, Heating and Photon Production from Arbitrary Energy Injections, Phys. Rev. D 93 (2016) 023521 [1506.03812].

[36] B. Dasgupta, R. Laha and A. Ray, Neutrino and positron constraints on spinning primordial black hole dark matter, Phys. Rev. Lett. 125 (2020) 101101 [1912.01014].

[37] S. Wang, D.-M. Xia, X. Zhang, S. Zhou and Z. Chang, Constraining the Primordial Black Holes as Dark Matter at JUNO, 2010.16053.

[38] R. Laha, Primordial Black Holes as a Dark Matter Candidate Are Severely Constrained by the Galactic Center 511 keV $\gamma$-Ray Line, Phys. Rev. Lett. 123 (2019) 251101 [1906. 09994].

[39] R. Laha, J.B. Muñoz and T.R. Slatyer, INTEGRAL constraints on primordial black holes and particle dark matter, Phys. Rev. D 101 (2020) 123514 [2004.00627].

[40] W. DeRocco and P.W. Graham, Constraining Primordial Black Hole Abundance with the Galactic 511 keV Line, Phys. Rev. Lett. 123 (2019) 251102 [1906.07740].

[41] N. Prantzos et al., The $511 \mathrm{keV}$ emission from positron annihilation in the Galaxy, Rev. Mod. Phys. 83 (2011) 1001 [1009.4620].

[42] C.A. Kierans et al., Positron Annihilation in the Galaxy, 1903.05569.

[43] S. Clark, B. Dutta, Y. Gao, Y.-Z. Ma and L.E. Strigari, $21 \mathrm{~cm}$ limits on decaying dark matter and primordial black holes, Phys. Rev. D 98 (2018) 043006 [1803.09390].

[44] J.D. Bowman, A.E.E. Rogers, R.A. Monsalve, T.J. Mozdzen and N. Mahesh, An absorption profile centred at 78 megahertz in the sky-averaged spectrum, Nature 555 (2018) 67 [1810.05912].

[45] CORE collaboration, Exploring cosmic origins with CORE: Cosmological parameters, JCAP 04 (2018) 017 [1612.00021].

[46] CORE collaboration, Exploring cosmic origins with CORE: Survey requirements and mission design, JCAP 04 (2018) 014 [1706.04516].

[47] M. Hazumi, P. Ade, Y. Akiba, D. Alonso, K. Arnold, J. Aumont et al., Litebird: A satellite for the studies of b-mode polarization and inflation from cosmic background radiation detection, Journal of Low Temperature Physics 194 (2019) .

[48] M. Alvarez et al., PICO: Probe of Inflation and Cosmic Origins, 1908.07495.

[49] NASA PICO collaboration, PICO: Probe of Inflation and Cosmic Origins, 1902.10541.

[50] K. Young et al., Optical Design of PICO, a Concept for a Space Mission to Probe Inflation and Cosmic Origins, 8, 2018 [1808.01369]. 
[51] E. Calabrese et al., Precision Epoch of Reionization studies with next-generation CMB experiments, JCAP 1408 (2014) 010 [1406.4794].

[52] Y.-P. Li, Y. Liu, S.-Y. Li, H. Li and X. Zhang, Tibet's Ali: A New Window to Detect the CMB Polarization, 1709.09053.

[53] H. Li, S.-Y. Li, Y. Liu, Y.-P. Li and X. Zhang, Tibet's window on primordial gravitational waves, Nature Astron. 2 (2018) 104 [1802.08455].

[54] K. Arnold et al., The Simons Array: expanding POLARBEAR to three multi-chroic telescopes, Proc. SPIE Int. Soc. Opt. Eng. 9153 (2014) 91531F.

[55] P. Creminelli, D.L. López Nacir, M. Simonović, G. Trevisan and M. Zaldarriaga, Detecting Primordial B-Modes after Planck, JCAP 1511 (2015) 031 [1502.01983].

[56] SPT collaboration, SPT-3G: A Multichroic Receiver for the South Pole Telescope, J. Low Temp. Phys. 193 (2018) 1057.

[57] K. Abazajian et al., CMB-S4 Science Case, Reference Design, and Project Plan, 1907.04473.

[58] CMB-S4 collaboration, CMB-S4 Science Book, First Edition, 1610.02743.

[59] P. Stöcker, M. Krämer, J. Lesgourgues and V. Poulin, Exotic energy injection with ExoCLASS: Application to the Higgs portal model and evaporating black holes, JCAP 03 (2018) 018 [1801.01871].

[60] S.K. Acharya and R. Khatri, $C M B$ and BBN constraints on evaporating primordial black holes revisited, JCAP 06 (2020) 018 [2002.00898].

[61] S.W. Hawking, Particle creation by black holes, Communications in Mathematical Physics 43 (1975) 199.

[62] J. MacGibbon and B. Webber, Quark and gluon jet emission from primordial black holes: The instantaneous spectra, Phys. Rev. D 41 (1990) 3052.

[63] T. Ukwatta, D. Stump, J. Linnemann, J. MacGibbon, S. Marinelli, T. Yapici et al., Primordial Black Holes: Observational Characteristics of The Final Evaporation, Astropart. Phys. 80 (2016) 90 [1510.04372].

[64] Jane, H. and MacGibbon, Quark- and gluon-jet emission from primordial black holes. ii. the emission over the black-hole lifetime, Physical Review D 44 (1991) 376-392.

[65] J. Cang, Y. Gao and Y.-Z. Ma, Probing Dark Matter with Future CMB Measurements, 2002.03380.

[66] S. Pi, Y.-l. Zhang, Q.-G. Huang and M. Sasaki, Scalaron from $R^{2}$-gravity as a heavy field, JCAP 05 (2018) 042 [1712.09896].

[67] A. Dolgov and J. Silk, Baryon isocurvature fluctuations at small scales and baryonic dark matter, Phys. Rev. D 47 (1993) 4244.

[68] B. Carr, K. Kohri, Y. Sendouda and J. Yokoyama, Constraints on primordial black holes from the Galactic gamma-ray background, Phys. Rev. D 94 (2016) 044029 [1604.05349].

[69] S. Clesse and J. García-Bellido, Massive Primordial Black Holes from Hybrid Inflation as Dark Matter and the seeds of Galaxies, Phys. Rev. D 92 (2015) 023524 [1501.07565].

[70] K. Kannike, L. Marzola, M. Raidal and H. Veermäe, Single Field Double Inflation and Primordial Black Holes, JCAP 09 (2017) 020 [1705.06225].

[71] J. Yokoyama, Cosmological constraints on primordial black holes produced in the near critical gravitational collapse, Phys. Rev. D 58 (1998) 107502 [gr-qc/9804041].

[72] J.C. Niemeyer and K. Jedamzik, Dynamics of primordial black hole formation, Phys. Rev. D 59 (1999) 124013 [astro-ph/9901292]. 
[73] B. Carr, M. Raidal, T. Tenkanen, V. Vaskonen and H. Veermäe, Primordial black hole constraints for extended mass functions, Phys. Rev. D 96 (2017) 023514 [1705.05567].

[74] A.M. Green, Microlensing and dynamical constraints on primordial black hole dark matter with an extended mass function, Phys. Rev. D 94 (2016) 063530 [1609.01143].

[75] N. Bellomo, J.L. Bernal, A. Raccanelli and L. Verde, Primordial Black Holes as Dark Matter: Converting Constraints from Monochromatic to Extended Mass Distributions, JCAP 01 (2018) 004 [1709.07467].

[76] B. Carr, T. Tenkanen and V. Vaskonen, Primordial black holes from inflaton and spectator field perturbations in a matter-dominated era, Phys. Rev. D 96 (2017) 063507 [1706.03746].

[77] X.-L. Chen and M. Kamionkowski, Particle decays during the cosmic dark ages, Phys. Rev. D 70 (2004) 043502 [astro-ph/0310473].

[78] H. Liu, T.R. Slatyer and J. Zavala, Contributions to cosmic reionization from dark matter annihilation and decay, Phys. Rev. D 94 (2016) 063507 [1604.02457].

[79] Y. Ali-Haimoud and C.M. Hirata, HyRec: A fast and highly accurate primordial hydrogen and helium recombination code, Phys. Rev. D 83 (2011) 043513 [1011.3758].

[80] J. Chluba, J.A. Rubino-Martin and R.A. Sunyaev, Cosmological hydrogen recombination: Populations of the high level sub-states, Mon. Not. Roy. Astron. Soc. 374 (2007) 1310 [astro-ph/0608242].

[81] J. Chluba, G.M. Vasil and L.J. Dursi, Recombinations to the Rydberg States of Hydrogen and Their Effect During the Cosmological Recombination Epoch, Mon. Not. Roy. Astron. Soc. 407 (2010) 599 [1003.4928].

[82] J. Chluba, D. Paoletti, F. Finelli and J.-A. Rubiño Martín, Effect of primordial magnetic fields on the ionization history, Mon. Not. Roy. Astron. Soc. 451 (2015) 2244 [1503.04827].

[83] J.A. Adams, S. Sarkar and D. Sciama, CMB anisotropy in the decaying neutrino cosmology, Mon. Not. Roy. Astron. Soc. 301 (1998) 210 [astro-ph/9805108].

[84] L. Lopez-Honorez, O. Mena, A. Moliné, S. Palomares-Ruiz and A.C. Vincent, The $21 \mathrm{~cm}$ signal and the interplay between dark matter annihilations and astrophysical processes, JCAP 08 (2016) 004 [1603.06795].

[85] G. Mellema et al., Reionization and the Cosmic Dawn with the Square Kilometre Array, Exper. Astron. 36 (2013) 235 [1210.0197].

[86] C.M. Trott and J.C. Pober, The status of 21cm interferometric experiments, 1909.12491.

[87] Simons ObSERvatory collaboration, The Simons Observatory: Science goals and forecasts, JCAP 1902 (2019) 056 [1808.07445].

[88] A. Lewis, A. Challinor and A. Lasenby, Efficient computation of CMB anisotropies in closed FRW models, Astrophys. J. 538 (2000) 473 [astro-ph/9911177].

[89] Planck collaboration, Planck 2018 results. VI. Cosmological parameters, 1807.06209.

[90] J. Errard, S.M. Feeney, H.V. Peiris and A.H. Jaffe, Robust forecasts on fundamental physics from the foreground-obscured, gravitationally-lensed CMB polarization, JCAP 03 (2016) 052 [1509.06770].

[91] S. Hamimeche and A. Lewis, Likelihood Analysis of CMB Temperature and Polarization Power Spectra, Phys. Rev. D77 (2008) 103013 [0801.0554].

[92] PLANCK collaboration, Planck 2018 results. V. CMB power spectra and likelihoods, 1907.12875.

[93] A. Lewis and S. Bridle, Cosmological parameters from CMB and other data: A Monte Carlo approach, Phys. Rev. D66 (2002) 103511 [astro-ph/0205436]. 
[94] A. Lewis, Efficient sampling of fast and slow cosmological parameters, Phys. Rev. D87 (2013) 103529 [1304.4473].

[95] F. Kühnel and K. Freese, Constraints on Primordial Black Holes with Extended Mass Functions, Phys. Rev. D 95 (2017) 083508 [1701.07223]. 